Abstract ID: 115

\title{
The reliability of an error classification system developed for use by the International Islamic University Malaysia Primary Care Clinic
}

\author{
Nuur Asikin Abd Raman ${ }^{\mathrm{a}} \mid$ Samsul Draman ${ }^{\mathrm{b}}$ | Ibrahim Adham Taib ${ }^{\mathrm{a}}$ \\ ${ }^{a}$ Department of Biomedical Science, Kulliyyah Allied Health Sciences, International Islamic University \\ Malaysia \\ ${ }^{b}$ Department of Family Medicine, Kulliyyah Medicine, International Islamic University Malaysia
}

Introduction: Primary care is often the entry point for patients in a healthcare system. The literature, however suggests that primary care is not as safe as it should be due to medical errors. Because no error classification has been developed for preventing medical errors in Malaysian primary care clinics. Thus, this study aimed to develop an error classification system for use in the International Islamic University Malaysia Primary Care Clinic (IIUMPC) before assessing its reliability and acceptability to potential users. Methods: An error classification system for primary care was developed by merging four existing error classification systems. This cross-sectional study, then recruited 107 healthcare students as participants. Each student had to read 3 case studies on medical errors in primary care before classifying each case using the newly developed error classification system. The students then had to fill in a questionnaire to provide feedback about the classification system. The interrater reliability was then compared using Krippendorff's alpha. Results: Krippendorff's alpha coefficient computed for Case I was 0.358, Case II was 0.369, while Case III was 0.314. The mean Krippendorff's alpha coefficient for all three cases was 0.347 . $72 \%$ of participants indicated their willingness to use the classification system in the future, citing ease of use (48\%) among others. Conclusions: Although a new error classification system was successfully developed for use in the IIUMPC, the level of agreement between classifiers was considered low. Nevertheless, the positive responses by the participants warrant the classifications system's improvement.

KEYWORDS: Error classification system, medical error, patient safety, primary care 